to admit the tentative nature of scientific hypotheses. Sciences should be taught inductively, i.e., with reference to the history of the mental processes and environments of its founders. Most hypotheses are private in origin but democratic in acceptance. The category of truth or falsity does not apply to hypotheses. It is therefore a fallacy to assume that all propositions must either be true or be false. In respect of truth and falsity there are (at least) the following classes of propositions.

1. Propositions about empirical public facts. A further appeal to experience can demonstrate the truth of these.

2. Propositions about historic facts whose truth is decided by the consensus of historical opinion.

3. Propositions about legal facts whose truth is decided by a court of law.

4. Propositions about private facts. As to the truth of these the individual is the sole judge.

5. Hypothetical propositions. These are all based on analogy and are therefore neither true nor untrue. They are either more or less convenient for the purpose in hand. Mathematical and geometric propositions belong here too, for metrical mathematics judges usefulness by the mutual consistency of statements made within a given conventional system, while geometric propositions are based on axioms and postulates the reliability of which is finally tested by experience.

\title{
Quantum Theory and Logic
}

\author{
BY DR. M. D. H. STRAUSS
}

ABSTRACT of Paper read on 20th February, 1950

The continued discussion about the so-called logical problems of quantum theory (QT) [cf., e.g., Reichenbach's book, recent issues of Dialectica and Philosophy of Science] is due to the absence of a recognized physical axiomatics to which these problems could be referred for settlement. The failure of various attempts to re-construct $\mathrm{QT}$ on the basis of simple physical postulates is a sign that a complete understanding of QT has not yet been achieved, or else that something entirely new is involved in such a reconstruction.

The reconstruction of the basic features of mathematical QT, carried out in 1936 by Dr. Strauss, $\S$ is based on what may be described as a translation of Bohr's conception of complementarity into the language of logical syntax where it gives rise to a new "logic" characterized by restricted sentential connectability. The main contentions of Dr. Strauss' lecture were (i) that this complementarity logic is absolutely essential in any rational reconstruction of QT, and (ii) that it accounts for the most general features of our experience in the field of quantum physics in exactly the same sense in which nonEuclidean geometry accounts for the basic feature of gravitation, i.e. the equivalence of gravitation and acceleration, or in which the Lorentz-transformation accounts for the basic fact in kinematics, viz., the existence of a finite limiting velocity.

The "most general feature of quantum physical experience" may be extracted either from an analysis of QT itself or from that of typical quantum phenomena. It consists (i) in the statistical character of all well-defined predictions, and (ii) in the relationship of general complementarity which holds between the results of any two actual measurements or observations (other than those of a constant quantity), and which may be stated thus : the results

§ Zur Begruendung der Statistischen Transformationstheorie der Quantenphysik,

Ber, Berl. Akad., phys,-math. Kl., Vol, XXVII (1936), 
of two measurements cannot be used simultaneously in the same prognostic or retrospective argument if physically correct conclusions are to be drawn. In the non-relativistic theory this reduces to the simpler statement that of two consecutive measurements only the first can be used for a retrospective analysis while only the second can be used for prognosis. The much discussed impossibility of simultaneous measurement and indeed of the definition of simultaneous attributes (special complementarity), far from being the root of indeterminacy in quantum physics, turns out to be a logical consequence of the relationship of general complementarity.

In discussing methods of theory construction Dr. Strauss had already suggested that the secret of progress in theoretical physics lies in the choice of a technical language which by its very syntax accounts for the most general features of our physical experience, a secret first revealed in the construction of Einstein's theory of gravitation. Using this method, and accordingly formulating the relationship of general complementarity as a syntactic rule for the quantum theoretical language, one obtains directly the above-mentioned " complementarity logic" (restricted sentential connectability) the calculus of which is isomorphic with the calculus of projection operators used in the general formulation of QT. Moreover, a consideration of compound probability expressions such as occur in the general theorem for the multiplication of probabilities then shows that the projection operators have to be Hermitean (and not real) since otherwise the mathematical theory would give an answer to questions to which, according to the principle of general complementarity, there is no definite answer in terms of probabilities. The use of complex-valued probability amplitudes in the Schroedinger representation of the theory is thus shown to be a direct consequence of the principle of complementarity.

While it would be tempting to discuss the ideas advanced by other workers in this field the results of such a discussion would have an essentially negative character since none of these ideas is powerful enough to result in a reconstruction of QT on an empirical basis.

The reconstruction of $\mathrm{QT}$ outlined above leads to a separation of those features of present QT which are fundamental (projection operators in a space of unitary metric) and those which could be changed without changing the foundations (existence of a Hamiltonian, localizable fields) and which at present are determined by the principle of correspondence. [Among the quantum theories generally accepted at present the QT of the spinor fields is the only one which goes beyond this principle. However, it does not violate this principle since the latter is inapplicable to spinor fields (the notion of a spinor has no place in classical theory).] Unless the principle of correspondence is ill-applied in present QT, a break with the restrictive requirements of this principle appears to be necessary for any substantial progress in the theory of elementary particles. On the other hand, the principle of complementarity, and with it the fundamental features of mathematical QT, do not appear capable of any generalization and must therefore be expected to stay.

From the general point of view of theoretical logic (theory of theory construction), the most interesting feature of QT is perhaps not that it involves a sentential calculus different from any one invented by the professional logicians, but rather that this calculus together with its interpreting language [i.e., " the language of experimental physics supplemented with the terminology of classical theory" (Bohr)] constitutes a language-system of a syntactosemantic type more general than any of the types considered hitherto. Indeed, only in the case of special complementarity would the conjunction of two inconnectable sentences represent a meaningless statement, while in the case of general complementarity the rule of inconnectability cannot be " justified" 
on such simple grounds of semantics. By implication, then, Carnap's principle of tolerance has been extended from the realm of syntax to the wider field of syntacto-semantics.

In his concluding remarks Dr. Strauss expressed the opinion that the task of the logic of science lies in the future rather than in the past, and that the theory of theory construction, in its relation to theoretical physics, will develop in a way similar to the way in which theoretical physics itself has developed in relation to experimental physics:-from a tool of analysis and synthesis post factum through a period of close mutual collaboration to an operative rôle in the progress of science.

\section{Operational Analysis and the Nature of Some Physical Concepts}

'I'he following Report by Dr. G. J. Whitrow of the Meeting held on May 5, 1950, appeared in Nature of July 15, 1950. It is reproduced here by kind permission of the Editors of Nature.

The Philosophy of Science Group of the British Society for the History of Science, under the chairmanship of Prof. J. H. Woodger, held a meeting on 5th May at University College, London. A general discussion took place on questions arising out of Prof. P. W. Bridgman's three lectures on "The Nature of Some of our Physical Concepts" delivered at University College during April 24-28. (These lectures are to be published in the British Journal for the Philosophy of Science.) The meeting was attended by about forty members of the Group and visitors.

Prof. Bridgman opened the discussion with a brief summary of his lectures. He thought that their most important feature was the detailed analysis of how the operational technique can be applied to specific physical concepts and problems. In the first lecture, he had discussed general concepts such as field, action at a distance, and empty space; in the second, thermodynamical phenomena, in particular the laws relating to energy and entropy ; and in the third, some situations presented by thermo-electric phenomena. Prof. Bridgman believed that the particular novelty of his analysis in these lectures was the self-conscious separation of physical operations into those of an instrumental character and those of a "paper-and-pencil "- that is, mentalcharacter. He had been aware of this distinction for a long time, but only recently had he seen how it applied to what physicists actually do. With regard to this, there are two points of view, as follows.

Instrumental operations correspond most nearly to what used to be called "physical reality". This aspect of physics can be illustrated by an analysis of the field concept. Instrumentally, the existence of a field at a certain place means that, if you go there with the appropriate type of instrument, it will record a certain " reading". Similarly, in applying the first law of thermodynamics, one must specify the elements of space, and in particular their boundaries, on which one operates, so that the fluxes over these boundaries correspond to the actual instrumental readings.

The other aspect relates to the way in which our verbal demands interlock with our instrumental operations. For example, we accept the typical conservation laws of physics as verbal guides with rational implications, but we also find that there exist instrumental operations corresponding to our verbal demands.

It is Prof. Bridgman's belief that, in general, no sharp dividing line can be drawn in physics between the purely instrumental and the purely mental. He regards the whole operational approach as part of a larger programmo, according to which we regard the world about us in terms of "activity" rather 